\title{
Modulating P2X7 Receptor Signaling during Rheumatoid Arthritis: New Therapeutic Approaches for Bisphosphonates
}

\author{
Alberto Baroja-Mazo and Pablo Pelegrín \\ Inflammation and Experimental Surgery Unit, CIBERehd, University Hospital "Virgen de la Arrixaca" and Foundation for \\ Healthcare Training and Research of The Region of Murcia (FFIS), Carretera Madrid-Cartagena s/n, 30120 Murcia, Spain \\ Correspondence should be addressed to Alberto Baroja-Mazo, alberto.baroja@ffis.es
}

Received 29 February 2012; Revised 28 May 2012; Accepted 30 May 2012

Academic Editor: Niklas Rye Jørgensen

Copyright ( 2012 A. Baroja-Mazo and P. Pelegrín. This is an open access article distributed under the Creative Commons Attribution License, which permits unrestricted use, distribution, and reproduction in any medium, provided the original work is properly cited.

\begin{abstract}
$\mathrm{P} 2 \mathrm{X} 7$ receptor-mediated purinergic signaling is a well-known mechanism involved in bone remodeling. The P2X7 receptor has been implicated in the pathophysiology of various bone and cartilage diseases, including rheumatoid arthritis (RA), a widespread and complex chronic inflammatory disorder. The P2X7 receptor induces the release into the synovial fluid of the proinflammatory factors (e.g., interleukin- $\beta$, prostaglandins, and proteases) responsible for the clinical symptoms of RA. Thus, the P2X7 receptor is emerging as a novel anti-inflammatory therapeutic target, and various selective P2X7 receptor antagonists are under clinical trials. Extracellular ATP signaling acting through the P2X7 receptor is a complex and dynamic scenario, which varies over the course of inflammation. This signaling is partially modulated by the activity of ectonucleotidases, which degrade extracellular ATP to generate other active molecules such as adenosine or pyrophosphates. Recent evidence suggests differential extracellular metabolism of ATP during the resolution of inflammation to generate pyrophosphates. Extracellular pyrophosphate dampens proinflammatory signaling by promoting alternative macrophage activation. Our paper shows that bisphosphonates are metabolically stable pyrophosphate analogues that are able to mimic the anti-inflammatory function of pyrophosphates. Bisphosphonates are arising per se as promising anti-inflammatory drugs to treat RA, and this therapy could be improved when administrated in combination with $\mathrm{P} 2 \mathrm{X} 7$ receptor antagonists.
\end{abstract}

\section{Introduction}

Rheumatoid arthritis (RA) is a widespread chronic systemic inflammatory disorder that affects approximately $1 \%$ of the worldwide population. The female-to-male ratio of the disease is $3: 1$, and although it can occur at any age, it is more common between ages 40 and 70 years [1]. In this context, pharmaceutical companies are interested in developing new anti-inflammatory treatments for the disease, including the use of P2X7 receptor antagonists or bisphosphonates [2, 3]. This paper will focus on the role of $\mathrm{P} 2 \mathrm{X} 7$ receptors in the pathophysiology of RA and the possible therapeutic connection of bisphosphonates with $\mathrm{P} 2 \mathrm{X} 7$ receptor signaling.

\section{P2X7 Receptor in Bone and Cartilage}

Bone is a specialized connective tissue composed of mineralized extracellular matrix and distinct cell populations including osteoblasts, osteocytes, and osteoclasts. Under physiological conditions, bone is subjected to a continuous balance between resorption and formation. However, disturbances of this balance can lead to various diseases such as osteoporosis, RA, or periodontitis [4]. The balance is regulated in bone by a complex network of factors, including hormones and mechanical stimulation. The latter, in turn, induces nucleotide release to the extracellular space and purinergic P2-receptor signaling [5]. P2 receptors are expressed in a variety of cell types in the bone and cartilage, including osteoblasts, osteoclasts, chondrocytes, and synoviocytes and are subdivided into two classes: the P2Y family of G-protein-coupled receptors and the P2X family of ligand-gated cation channels [6].

Recent evidence reviewed by Grol et al. [7] provides specific insight into the role of the $\mathrm{P} 2 \mathrm{X} 7$ receptor subtype in osteoblasts and osteoclasts. Additionally, P2X7 receptor 
knockout mice exhibit decreased periosteal bone formation, increased trabecular bone resorption, and impaired response to mechanical stimulation, leading to a reduction in total bone content $[8,9]$. P2X7 receptor activation in osteoblasts enhances differentiation and bone formation [10], whereas its activation in osteoclasts results in apoptosis [11]. These differences in the function of $\mathrm{P} 2 \mathrm{X} 7$ receptor reflect a sophisticated mechanism whereby the skeleton responds to mechanical stimulation by simultaneously increasing bone formation and suppressing its resorption. Furthermore, genetic loss-of-function polymorphisms of the human P2X7 receptor are related with increased skeletal fragility, which is consistent with decreased susceptibility of osteoclasts to apoptosis, as well as impaired osteoblast differentiation and bone formation $[12,13]$. P2X7 receptor modulation could also play an important role in regulating bone-cell response, and ATP appears to mediate internalization of $\mathrm{P} 2 \mathrm{X} 7$ receptors in osteoclast-like cells [14].

Mechanical stimulation of different cell types, including osteoblasts and chondrocytes, induces ATP release through hemichannel opening [15-17]. Osteoblast-like cells constitutively release nucleotides into the extracellular environment [18]. ATP released into the extracellular compartment of the bone could activate $\mathrm{P} 2 \mathrm{X} 7$ receptors on osteoblasts and osteoclasts and the function of $\mathrm{P} 2 \mathrm{X} 7$ receptor in bone is consistent with the altered skeleton phenotype of $\mathrm{P} 2 \mathrm{X} 7$ receptor knockout mice described by Ke et al. [8]. In this regard, pores formed in response to $\mathrm{P} 2 \mathrm{X} 7$ receptor activation induces additional ATP release, initiating a positive purinergic feedback loop [19]. Although the physiological importance of this phenomenon remains unknown, in vitro mechanical stimulation of osteoblasts leads to cell permeabilization via a mechanism dependent on P2-receptor signaling [9].

\section{P2X7 Receptor in the Pathophysiology of RA}

Purinergic signaling has been implicated in the pathophysiology of various bone and cartilage diseases, including bone loss, RA, osteoarthritis, and bone cancer pain [2024]. RA is a widespread and complex chronic inflammatory disorder with no current successful treatment [1]. Because it is a complex multifactorial disease, its pathophysiology is not fully understood; however, there is evidence to suggest that $\mathrm{T}$ lymphocytes and macrophages play a critical role in the initiation and perpetuation of synovial inflammation [25]. Interleukin (IL) $-1 \beta$ and tumor necrosis factor (TNF)- $\alpha$ are macrophage-derived cytokines that play a primary role in the pathogenesis of RA. One effect of these cytokines is to regulate the production of matrix metalloproteinases (MMPs), which are directly involved in extracellular matrix degradation during RA [26]. In fact, the serum and synovial concentrations of TNF- $\alpha$ and IL- $1 \beta$ are high in patients with active RA [27], and drugs targeting TNF- $\alpha$ were the first biologics to be approved and widely used to treat RA. At present, five TNF inhibitors are approved for use by the U.S. Food and Drug Administration; all of these agents have been shown to be effective in reducing the clinical signs of inflammation in RA patients [27].
Experimental injection of IL- $1 \beta$ into the knee joints of rabbits resulted, within hours, in leukocyte accumulation in the synovial fluid as well as substantial proteoglycan loss from joint cartilage $[28,29]$. Unlike $\mathrm{TNF}-\alpha$, which is predominantly detected in the early stages of disease, IL- $1 \beta$ is detected long after the onset of RA [30]. IL-1 $\beta$ is a potent stimulator of leukocytic infiltration, synovial hyperplasia, cell activation, cartilage breakdown, and inhibition of cartilage matrix synthesis [31].

A two-step process tightly regulates IL- $1 \beta$ production. The first step regulates the synthesis of IL- $1 \beta$ precursor (pro-IL-1 $\beta$ ) by engagement of innate pattern recognition receptors such as toll-like receptors (TLR), activating nuclear factor $-\kappa \mathrm{B}(\mathrm{NF}-\kappa \mathrm{B})$, and mitogen-activated protein kinase (MAPK) transcription pathways [32]. The second stimulation signal leads to the formation of a multiprotein complex called the inflammasome, which culminates in caspase- 1 activation and pro-IL- $1 \beta$ maturation. The bestcharacterized inflammasome is formed by the NLRP3 receptor (nucleotide-binding domain and leucine-rich repeat receptor containing pyrin domain 3 ), the adaptor protein ASC (apoptotic speck-like protein with a caspase-activating recruiting domain), and caspase-1. Numerous stimuli trigger the NLRP3 inflammasome, including pathogen- or dangerassociated molecular patterns (PAMPs and DAMPs, resp.) [33]. Extracellular ATP activating P2X7 receptor is one of the most widely investigated DAMPs that activate the NLRP3 inflammasome and is probably one of the most important pathways for IL- $1 \beta$ release in RA. Extracellular ATP is found at high concentrations in the synovial fluid of patients with RA [34], and P2X7 receptor deficiency leads to a lower incidence and lower severity of joint inflammation in animal models of arthritis induced by anticollagen antibodies [35]. RA-associated synoviocytes express functional P2X7 receptors, and their activation upregulates the production of proinflammatory cytokine IL-6 from these cells [36].

Interest in developing new drugs that target the synthesis, processing, and/or release of IL-1 $\beta$ has risen in recent years [23]. Blocking antibodies for soluble IL-1 $\beta$ have been shown to reduce joint destruction in several animal models of RA [37]. In a phase II clinical trial with RA patients, 24 weeks of treatment with the recombinant IL1-receptor antagonist (IL-1Ra or anakinra) provided significantly greater clinical improvement of RA symptoms than placebo [38]. Anakinra treatment is safe and highly effective for patients with systemic-onset juvenile idiopathic arthritis, adult-onset Still's disease, hereditary autoinflammatory syndromes, Schnitzler's syndrome, and gouty attacks [39]. However, this treatment has been associated with liver toxicity, and long-term follow-up analyses are essential to guide appropriate management strategies [40]. Selective drug-like P2X7 receptor antagonists have already been tested in clinical trials for RA. Nonetheless, after initial positive outcomes in phase II trials, AstraZeneca and Pfizer discontinued the development of their compounds following negative phase IIb/III results in subjects receiving methotrexate [2, 41]. The poor pharmacokinetics and pharmacodynamics of AstraZeneca's and Pfizer's P2X7 receptor antagonists could partially explain the lack of effectiveness. However, 
as described above, the fact that RA pathophysiology could be strongly mediated by TNF- $\alpha$ might also explain such poor outcomes. Recently, a new generation of P2X7 receptor antagonists with better drug-like properties is entering earlyphase clinical studies [40].

New evidence suggests that $\mathrm{P} 2 \mathrm{X} 7$ receptor activation also triggers inflammasome- and cytokine-independent pathways that play an active role in the pathogenesis of RA. In fact, an effective anti-P2X7 receptor therapy would be more beneficial than anticytokine directed therapy. P2X7 receptor activation has been associated with the release of the tissuedestroying proteases MMPs and cathepsins [22, 42, 43]. Cathepsin is a family of lysosomal proteases implicated in cartilage joint destruction and found in the synovial fluid of patients with active RA [44, 45]. Treatment with P2X7 antagonist, but not with other active RA drugs such as methotrexate, abolished cathepsin release from ATPactivated macrophages [22]. P2X7 receptor activation has also been associated with the release of prostaglandin E2 (PGE2) and other autacoids [46]. In particular, PGE2 induces the release of MMPs in the joint during RA and is a key mediator of pain sensation [26].

\section{Bisphosphonates: Anti-Inflammatory Compounds in RA}

Because of their anti-inflammatory properties, bisphosphonates may represent a promising new drug for the treatment of RA. Bisphosphonates are metabolically stable analogues of pyrophosphate, where the central oxygen atom is replaced by a carbon atom (Figure 1). The R1 and R2 side chains bonded to the central carbon confer different properties to the bisphosphonate molecule, whereas R1 is usually short and is involved in binding to bone mineral and R2 is responsible for its biological effects $[47,48]$. Depending on the side-chain structure of R2, bisphosphonates are classified into nitrogen-containing bisphosphonates ( $N$ bisphosphonates, such as pamidronate, risedronate, ibandronate or zolendronate) and nonnitrogen-containing bisphosphonates (non- $N$-bisphosphonates, such as etidronate or clodronate) (Figure 1).

Bisphosphonates are able to bind divalent ions, such as $\mathrm{Ca}^{2+}$, and therefore target exposed bone mineral surfaces in vivo. After bone binding, bisphosphonates are incorporated in bone-resorbing osteoclasts, resulting in osteoclast function inhibition and apoptosis [49]. Bisphosphonates that selectively affect osteoclasts have become a major class of antiresorptive drug for the treatment of osteoporosis and Paget's disease $[50,51]$. Bisphosphonates can also be encapsulated in liposomes and then selectively used to target phagocytic cells in vivo [52]. Liposome-encapsulated clodronate is widely used to eliminate macrophages in vivo and has been shown to reduce RA-associated inflammation in animal models and in patients [3].

Several molecular mechanisms have been described for the action of bisphosphonates. Non- $N$-bisphosphonates can be metabolized to nonhydrolyzable and $\beta, \gamma$-methylenecontaining (AppCp-type) analogues of ATP [53]. Intracellular accumulation of these metabolites arrest osteoclast and
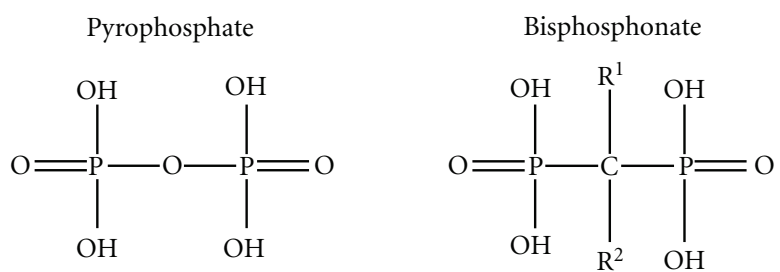

\begin{tabular}{lll} 
& $\mathrm{R}^{1}$ & \multicolumn{2}{l}{$\mathrm{R}^{2}$} \\
Etidronate & $\mathrm{OH}$ & $\mathrm{CH}_{3}$ \\
Clodronate & $\mathrm{Cl}$ & $\mathrm{Cl}$ \\
Pamidronate & $\mathrm{OH}$ & $\mathrm{CH}_{2} \mathrm{CH}_{2} \mathrm{NH}_{2}$ \\
Ibandronate & $\mathrm{OH}$ & $\mathrm{CH}_{2} \mathrm{CH}_{2} \mathrm{~N}\left(\mathrm{CH}_{3}\right)($ phenyl $)$ \\
Risedronate & $\mathrm{OH}$ & $\mathrm{CH}_{2}-3$-pyridine \\
Zoledronate & $\mathrm{OH}$ & $\mathrm{CH}_{2}(1 \mathrm{H}$-imidazole-1-y1)
\end{tabular}

Figure 1: Pyrophosphate and bisphosphonate structures.

macrophage metabolism and function. The in vivo accumulation of $\mathrm{AppCCl}_{2} \mathrm{p}$ in osteoclasts induces apoptosis and inhibits bone resorption [54, 55]. $N$-bisphosphonates are also known to affect normal cellular function by inhibiting farnesyl diphosphate synthase and thereby preventing the prenylation of small GTPases $[47,56]$.

Additionally, several studies demonstrate that bisphosphonates are antioxidant compounds. Reactive oxygen species (ROS) are known to contribute to the inflammatory process in RA by degrading cartilage and bone [57]. In an inflamed joint, ROS are produced by macrophages, neutrophils, and chondrocytes [58]. Serretti et al. [59] showed that bisphosphonates inhibit ROS production in human neutrophils by acting before or on NADPH oxidase. Bisphosphonates also have antioxidant properties as iron chelators and block chondrocyte lipid peroxidation, suggesting a protective role for bisphosphonates in RA [60].

\section{Bisphosphonates, Extracellular Metabolism of Nucleotides, and P2X7 Receptors}

Our group recently identified clodronate and its physiological analogue pyrophosphate as a new molecule structure able to block IL-1 $\beta$ release [61]. This inhibition was also found by using high ATP concentrations during macrophage polarization towards anti-inflammatory or alternatively activated M2 states, where P2X7 receptor signaling was uncoupled from inflammasome activation. In proinflammatory M1 macrophages, this effect was achieved when the P2X7 receptor was absent or pharmacologically blocked. We previously proposed a model for inflammasome regulation by pyrophosphates and extracellular ATP during macrophage polarization gradient towards M2 where P2X7 receptor uncouples from both ROS production and the NLRP3-inflammasome/caspase-1 pathway, while P2X7 receptor remains functional in terms of its ion channel activity [60]. Under 


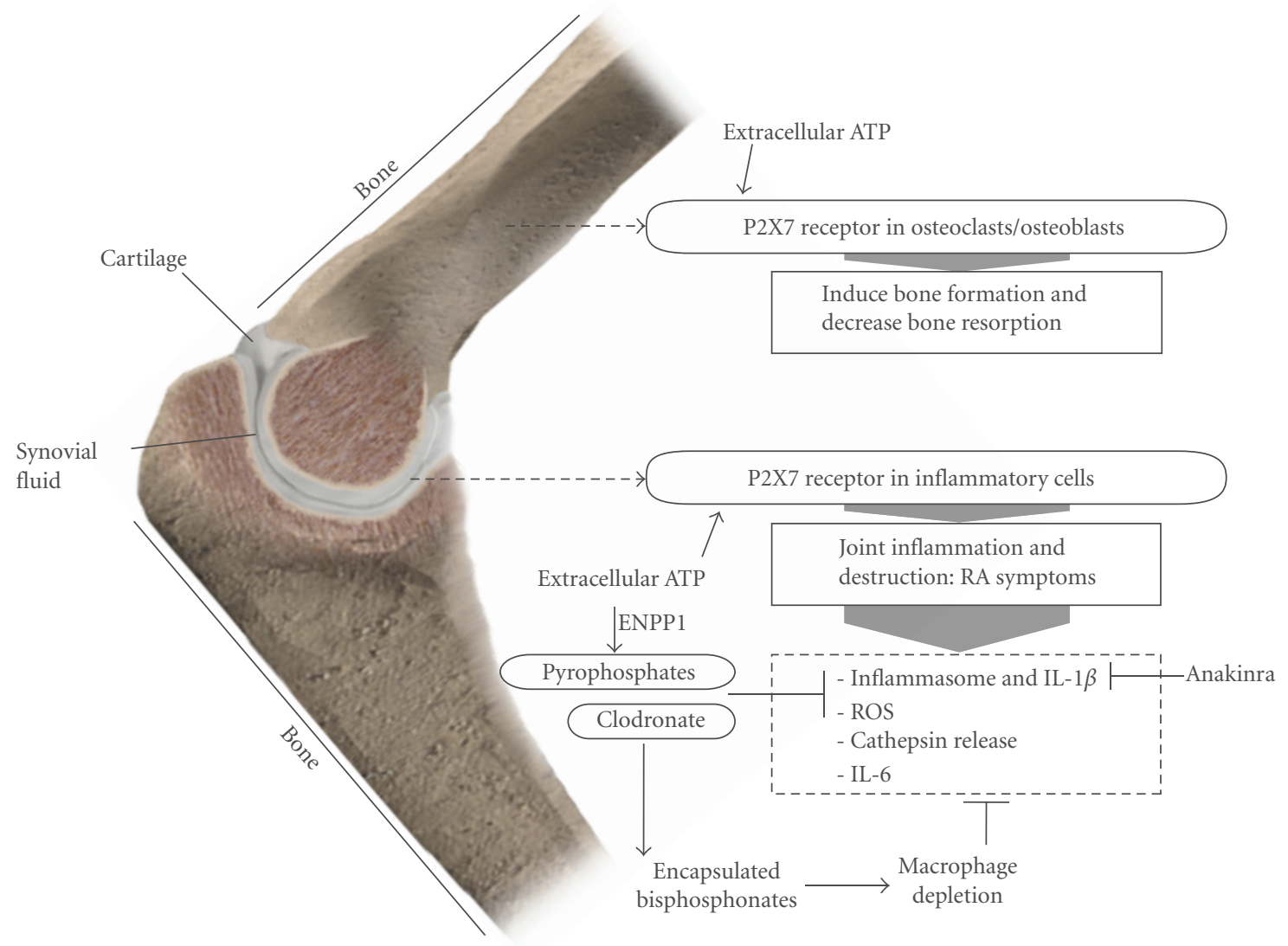

FIGURE 2: Role of extracellular ATP and P2X7 receptor in bone physiology and cartilage inflammation.

these conditions, pyrophosphates act to inhibit ROS and cluster actin dynamics induced by other inflammasome activators (e.g., maitotoxin), resulting in blockage of caspase-1 and IL- $1 \beta$ release. This effect could be beneficial in enhancing the resolving phase of inflammation.

Our evidence suggested that the diphosphate group resident in nucleotides was blocking inflammasome activation, because pyrophosphates and triphosphates but not monophosphates (inorganic phosphate) inhibited caspase-1 activation and IL- $1 \beta$ release, with a potency order of clodronate $>$ triphosphate $=$ diphosphate $>$ ATP $\gg$ ADP. It is still unknown if pyrophosphates act by phosphate chains remaining attached to the nucleotide molecule or if they result from ATP metabolism by ectonucleotidases. Ectonucleotidases might play a play in this process, because the expression of ectonucleotidases and other genes involved in the extracellular generation of pyrophosphate is highly expressed by M2 compared to M1 macrophages [62]. M2 macrophages accumulate the transcript for ectonucleotide pyrophosphatase/phosphodiesterase 1 (ENPP1), which primarily degrades ATP to produce AMP and pyrophosphate [63]. M2 macrophages also accumulate the transcript for ANK, a plasma-membrane protein involved in progressive ankylosis that mediates cytosolic pyrophosphate release into the extracellular space [64].

Pyrophosphates were also able to alter the LPS-induced proinflammatory gene expression profile in a similar manner to the action of IL-4 (cytokine responsible for polarizing macrophages to M2). Interestingly, pyrophosphates were also able to upregulate ENPP1 expression after LPS stimulation, suggesting that over the course of the inflammatory process, extracellular pyrophosphate production could promote the initiation of resolution by shifting macrophage polarization to M2. In M1 macrophages, pyrophosphate and clodronate significantly inhibited gene expression dependent on NF- $\kappa \mathrm{B}$ activation, such as the LPS-induced production of proinflammatory cytokines (IL- $1 \beta$, IL- 6 , and TNF- $\alpha$ ) and the recovery of $\mathrm{I} \kappa \mathrm{B} \alpha$ protein [65]. Redox balance has been extensively implicated in NF- $\kappa$ B activation [66], and it has been found that pyrophosphates, so as clodronate, chelating the actions of ROS, could specifically affect the nuclear actions of translocated NF- $\kappa$ B to dampen proinflammatory gene expression and enhance ENPP1 gene expression [62]. Additionally, pyrophosphates are able to enhance the resolution of peritonitis in vivo by reducing proinflammatory cytokine production [62].

Taken together, these results suggest that extracellular ATP and its metabolism to pyrophosphate are key triggers in the switch from a proinflammatory macrophage towards its alternative functions in the resolution of inflammation. Pharmacologically, this effect could be mimicked and enhanced by the use of bisphosphonates as anti-inflammatory compounds. 


\section{Conclusions}

Several approaches can be taken to reduce synovial inflammation in RA, and many of them use the $\mathrm{P} 2 \mathrm{X} 7$ receptor as a central signaling molecule. These approaches include direct P2X7 receptor antagonism and extracellular IL- $1 \beta$ blocking (Figure 2). In fact, there is increasing evidence that bisphosphonates may be useful as novel anti-inflammatory drugs in RA. These compounds present various modes of action; for example, they deplete macrophages when administered encapsulated in liposomes. In contrast, free bisphosphonates present pyrophosphate-like activities that chelate ROS and block inflammasome activation during the resolving phase of inflammation, when $\mathrm{P} 2 \mathrm{X} 7$ receptor stimulation is uncoupled from proinflammatory signaling or when the P2X7 receptor is pharmacologically inhibited. In the latter case, bisphosphonates produce a switch in $\mathrm{P} 2 \mathrm{X} 7$ receptor signaling and extracellular nucleotide metabolism to pyrophosphates during the resolution of inflammation $[61,62]$. Therefore, a combination of P2X7 receptor antagonists with bisphosphonates could be more successful in treating chronic inflammation in RA.

\section{Abbreviations}

ANK:

AppCp-type:

ASC:

DAMPs:

ENPP1:

IL:

IL-1Ra:

IL-1RI:

MAPK:

MMPs:

$\mathrm{NF}-\kappa \mathrm{B}$ :

NLRP3:

$N$-bisphosphonates:

non- $N$-bisphosphonates: Nonnitrogen-containing

bisphosphonates

PAMPs: $\quad$ Pathogen-associated molecular patterns

PGE2: $\quad$ Prostaglandin E2

RA: $\quad$ Rheumatoid arthritis

ROS: $\quad$ Reactive oxygen species

TNF- $\alpha$ Tumor necrosis factor- $\alpha$

TLR: Toll-like receptors.

\section{Acknowledgments}

Work at Pelegrín's Lab is supported by Grants from PN I+D+I 2008-2011-Instituto Salud Carlos III-FEDER
(EMER07/049 and PI09/0120) and Fundación Séneca (11922/PI/09), managed by Fundación Formación Investigación Sanitaria Región de Murcia (FFIS).

\section{References}

[1] Y. Alamanos, P. V. Voulgari, and A. A. Drosos, "Incidence and prevalence of rheumatoid arthritis, based on the 1987 American College of Rheumatology criteria: a systematic review," Seminars in Arthritis and Rheumatism, vol. 36, no. 3, pp. 182$188,2006$.

[2] S. D. Guile, L. Alcaraz, T. N. Birkinshaw et al., "Antagonists of the P2X7 receptor. From lead identification to drug development," Journal of Medicinal Chemistry, vol. 52, no. 10, pp. 3123-3141, 2009.

[3] W. P. Maksymowych, "Bisphosphonates—anti-inflammatoy properties," Current Medicinal Chemistry, vol. 1, pp. 15-28, 2002.

[4] D. V. Novack and S. L. Teitelbaum, "The osteoclast: friend or foe?" Annual Review of Pathology, vol. 3, pp. 457-484, 2008.

[5] A. G. Robling, A. B. Castillo, and C. H. Turner, "Biomechanical and molecular regulation of bone remodeling," Annual Review of Biomedical Engineering, vol. 8, pp. 455-498, 2006.

[6] G. Burnstock, "Purine and pyrimidine receptors," Cellular and Molecular Life Sciences, vol. 64, no. 12, pp. 1471-1483, 2007.

[7] M. W. Grol, N. Panupinthu, J. Korcok, S. M. Sims, and S. J. Dixon, "Expression, signaling, and function of $\mathrm{P} 2 \mathrm{X} 7$ receptors in bone," Purinergic Signalling, vol. 5, no. 2, pp. 205-221, 2009.

[8] H. Z. Ke, H. Qi, A. F. Weidema et al., "Deletion of the P2X7 nucleotide receptor reveals its regulatory roles in bone formation and resorption," Molecular Endocrinology, vol. 17, no. 7, pp. 1356-1367, 2003.

[9] J. Li, D. Liu, H. Z. Ke, R. L. Duncan, and C. H. Turner, “The P2X7 nucleotide receptor mediates skeletal mechanotransduction," Journal of Biological Chemistry, vol. 280, no. 52, pp. 42952-42959, 2005.

[10] N. Panupinthu, J. T. Rogers, L. Zhao et al., "P2X7 receptors on osteoblasts couple to production of lysophosphatidic acid: a signaling axis promoting osteogenesis," Journal of Cell Biology, vol. 181, no. 5, pp. 859-871, 2008.

[11] A. Gartland, K. A. Buckley, R. A. Hipskind, W. B. Bowler, and J. A. Gallagher, "P2 receptors in bone-modulation of osteoclast formation and activity via P2X7 activation," Critical Reviews in Eukaryotic Gene Expression, vol. 13, no. 2-4, pp. 237-242, 2003.

[12] A. Gartland, K. K. Skarratt, L. J. Hocking et al., "Polymorphisms in the P2X7 receptor gene are associated with low lumbar spine bone mineral density and accelerated bone loss in post-menopausal women," European Journal of Human Genetics, vol. 20, no. 5, pp. 559-564, 2012.

[13] N. R. Jørgensen, L. B. Husted, K. K. Skarratt et al., "Singlenucleotide polymorphisms in the $\mathrm{P} 2 \mathrm{X} 7$ receptor gene are associated with post-menopausal bone loss and vertebral fractures," European Journal of Human Genetics, vol. 20, no. 6, pp. 675-681, 2012.

[14] J. F. Hiken and T. H. Steinberg, "ATP downregulates P2X7 and inhibits osteoclast formation in RAW cells," American Journal of Physiology, vol. 287, no. 2, pp. C403-C412, 2004.

[15] D. C. Genetos, D. J. Geist, D. Liu, H. J. Donahue, and R. L. Duncan, "Fluid shear-induced ATP secretion mediates prostaglandin release in MC3T3-E1 osteoblasts," Journal of Bone and Mineral Research, vol. 20, no. 1, pp. 41-49, 2005. 
[16] T. Iwamoto, T. Nakamura, A. Doyle et al., "Pannexin 3 regulates intracellular ATP/cAMP levels and promotes chondrocyte differentiation," Journal of Biological Chemistry, vol. 285, no. 24, pp. 18948-18958, 2010.

[17] M. M. Knight, S. R. Mcglashan, M. Garcia, C. G. Jensen, and C. A. Poole, "Articular chondrocytes express connexin 43 hemichannels and $\mathrm{P} 2$ receptors-a putative mechanoreceptor complex involving the primary cilium?" Journal of Anatomy, vol. 214, no. 2, pp. 275-283, 2009.

[18] K. A. Buckley, S. L. Golding, J. M. Rice, J. P. Dillon, and J. A. Gallagher, "Release and interconversion of P2 receptor agonists by human osteoblast-like cells," The FASEB Journal, vol. 17, no. 11, pp. 1401-1410, 2003.

[19] P. Pellegatti, "A novel recombinant plasma membrane-targeted luciferase reveals a new pathway for ATP secretion," Molecular Biology of the Cell, vol. 16, no. 8, pp. 3659-3665, 2005.

[20] C. A. Dinarello, "Blocking IL-1 in systemic inflammation," Journal of Experimental Medicine, vol. 201, no. 9, pp. 13551359, 2005.

[21] R. R. Hansen, C. K. Nielsen, A. Nasser et al., "P2X7 receptordeficient mice are susceptible to bone cancer pain," Pain, vol. 152, no. 8, pp. 1766-1776, 2011.

[22] G. Lopez-Castejon, J. Theaker, P. Pelegrin, A. D. Clifton, M. Braddock, and A. Surprenant, "P2X7 receptor-mediated release of cathepsins from macrophages is a cytokine-independent mechanism potentially involved in joint diseases," Journal of Immunology, vol. 185, no. 4, pp. 2611-2619, 2010.

[23] P. Pelegrin, "Targeting interleukin-1 signaling in chronic inflammation: focus on $\mathrm{P} 2 \mathrm{X}(7)$ receptor and Pannexin-1," Drug News \& Perspectives, vol. 21, no. 8, pp. 424-433, 2008.

[24] A. Wesselius, M. J. L. Bours, A. Agrawal et al., "Role of purinergic receptor polymorphisms in human bone," Frontiers in Bioscience, vol. 16, no. 7, pp. 2572-2585, 2011.

[25] K. L. Sewell and D. E. Trentham, "Pathogenesis of rheumatoid arthritis," The Lancet, vol. 341, no. 8840, pp. 283-286, 1993.

[26] D. Burger, J. M. Dayer, G. Palmer, and C. Gabay, "Is IL-1 a good therapeutic target in the treatment of arthritis?" Best Practice and Research, vol. 20, no. 5, pp. 879-896, 2006.

[27] E. H. S. Choy and G. S. Panayi, "Cytokine pathways and joint inflamation in rheumatoid arthritis," The New England Journal of Medicine, vol. 344, no. 12, pp. 907-916, 2001.

[28] J. M. Dayer, B. De Rochemonteix, and B. Burrus, "Human recombinant interleukin 1 stimulates collagenase and prostaglandin E2 production by human synovial cells," Journal of Clinical Investigation, vol. 77, no. 2, pp. 645-648, 1986.

[29] B. Henderson and E. R. Pettipher, "Comparison of the in vivo inflammatory activities after intra-articular injection of natural and recombinant IL- $1 \alpha$ and IL- $1 \beta$ in the rabbit," Biochemical Pharmacology, vol. 37, no. 21, pp. 4171-4176, 1988.

[30] A. K. Ulfgren, L. Klareskog, and M. Lindberg, "An immunohistochemical analysis of cytokine expression in allergic and irritant contact dermatitis," Acta Dermato-Venereologica, vol. 80, no. 3, pp. 167-170, 2000.

[31] C. A. Dinarello, "Biologic basis for interleukin-1 in disease," Blood, vol. 87, no. 6, pp. 2095-2147, 1996.

[32] M. R. Heitmeier, A. L. Scarim, and J. A. Corbett, "Doublestranded RNA-induced inducible nitric-oxide synthase expression and interleukin-1 release by murine macrophages requires NF- $\kappa \mathrm{B}$ activation," Journal of Biological Chemistry, vol. 273, no. 24, pp. 15301-15307, 1998.
[33] C. Bryant and K. A. Fitzgerald, "Molecular mechanisms involved in inflammasome activation," Trends in Cell Biology, vol. 19, no. 9, pp. 455-464, 2009.

[34] L. M. Ryan, J. W. Rachow, and D. J. McCarty, "Synovial fluid ATP: a potential substrate for the production of inorganic pyrophosphate," Journal of Rheumatology, vol. 18, no. 5, pp. 716-720, 1991.

[35] J. M. Labasi, N. Petrushova, C. Donovan et al., "Absence of the P2X7 receptor alters leukocyte function and attenuates an inflammatory response," Journal of Immunology, vol. 168, no. 12, pp. 6436-6445, 2002.

[36] F. Caporali, P. L. Capecchi, A. Gamberucci et al., "Human rheumatoid synoviocytes express functional P2X7 receptors," Journal of Molecular Medicine, vol. 86, no. 8, pp. 937-949, 2008.

[37] S. B. Abramson and A. Amin, "Blocking the effects of IL-1 in rheumatoid arthritis protects bone and cartilage," Rheumatology, vol. 41, no. 9, pp. 972-980, 2002.

[38] B. Bresnihan, J. M. Alvaro-Gracia, M. Cobby et al., "Treatment of rheumatoid arthritis with recombinant human interleukin1 receptor antagonist," Arthritis and Rheumatism, vol. 41, no. 12, pp. 2196-2204, 1998.

[39] G. D. Kalliolias and S. N. C. Liossis, "The future of the IL1 receptor antagonist anakinra: from rheumatoid arthritis to adult-onset still's disease and systemic-onset juvenile idiopathic arthritis," Expert Opinion on Investigational Drugs, vol. 17, no. 3, pp. 349-359, 2008.

[40] M. Mahamid, R. Mader, and R. Safadi, "Hepatotoxicity of tocilizumab and anakinra in rheumatoid arthritis: management decisions," Clinical Pharmacology, vol. 3, no. 1, pp. 3943, 2011.

[41] N. Arulkumaran, R. J. Unwin, and F. W. K. Tam, "A potential therapeutic role for $\mathrm{P} 2 \mathrm{X} 7$ receptor $(\mathrm{P} 2 \mathrm{X} 7 \mathrm{R})$ antagonists in the treatment of inflammatory diseases," Expert Opinion on Investigational Drugs, vol. 20, no. 7, pp. 897-915, 2011.

[42] C. Andrei, C. Dazzi, L. Lotti, M. R. Torrisi, G. Chimini, and A. Rubartelli, "The secretory route of the leaderless protein interleukin $1 \beta$ involves exocytosis of endolysosome-related vesicles," Molecular Biology of the Cell, vol. 10, no. 5, pp. 14631475, 1999.

[43] B. J. Gu and J. S. Wiley, "Rapid ATP-induced release of matrix metalloproteinase 9 is mediated by the P2X7 receptor," Blood, vol. 107, no. 12, pp. 4946-4953, 2006.

[44] Y. Hashimoto, H. Kakegawa, Y. Narita et al., "Significance of cathepsin B accumulation in synovial fluid of rheumatoid arthritis," Biochemical and Biophysical Research Communications, vol. 283, no. 2, pp. 334-339, 2001.

[45] D. Wang and D. Brömme, "Drug delivery strategies for cathepsin inhibitors in joint diseases," Expert Opinion on Drug Delivery, vol. 2, no. 6, pp. 1015-1028, 2005.

[46] M. Barbera-Cremades, A. Baroja-Mazo, A. I. Gomez et al., "P2X7 receptor-stimulation causes fever via PGE2 and IL-1 $\beta$ release," The FASEB Journal, vol. 26, pp. 2951-2962, 2012.

[47] A. J. Roelofs, K. Thompson, S. Gordon et al., "Molecular mechanisms of action of bisphosphonates: current status," Clinical Cancer Research, vol. 12, no. 20, pp. 6222s-6230s, 2006.

[48] M. J. Rogers, J. C. Frith, S. P. Luckman et al., "Molecular mechanisms of action of bisphosphonates," Bone, vol. 24, supplement 5, pp. 73S-79S, 1999.

[49] M. J. Rogers, S. Gordon, H. L. Benford et al., "Cellular and molecular mechanisms of action of bisphosphonates," Cancer, vol. 88, supplement 12, pp. 2961-2978, 2000. 
[50] J. D. Adachi, W. P. Olszynski, D. A. Hanley et al., "Management of corticosteroid-induced osteoporosis," Seminars in Arthritis and Rheumatism, vol. 29, no. 4, pp. 228-251, 2000.

[51] E. S. Siris, "Goals of treatment for Paget's disease of bone," Journal of Bone and Mineral Research, vol. 14, supplement 2, pp. 49-52, 1999.

[52] N. Van Rooijen and A. Sanders, "Liposome mediated depletion of macropbages: mechanism of action, preparation of liposomes and applications," Journal of Immunological Methods, vol. 174, no. 1-2, pp. 83-93, 1994.

[53] J. C. Frith, J. Mönkkönen, G. M. Blackburn, R. G. G. Russell, and M. J. Rogers, "Clodronate and liposome-encapsulated clodronate are metabolized to a toxic ATP analog, adenosine 5'-(beta, gamma-dichloromethylene) triphosphate, by mammalian cells in vitro," Journal of Bone and Mineral Research, vol. 12, no. 9, pp. 1358-1367, 1997.

[54] J. C. Frith, J. Mönkkönen, S. Auriola, H. Mönkkönen, and M. J. Rogers, "The molecular mechanism of action of the antiresorptive and antiinflammatory drug clodronate: evidence for the formation in vivo of a metabolite that inhibits bone resorption and causes osteoclast and macrophage apoptosis," Arthritis and Rheumatism, vol. 44, no. 9, pp. 2201-2210, 2001.

[55] P. P. Lehenkari, M. Kellinsalmi, J. P. Näpänkangas et al., "Further insight into mechanism of action of clodronate: inhibition of mitochondrial ADP/ATP translocase by a nonhydrolyzable, adenine-containing metabolite," Molecular Pharmacology, vol. 61, no. 5, pp. 1255-1262, 2002.

[56] F. P. Coxon, K. Thompson, and M. J. Rogers, "Recent advances in understanding the mechanism of action of bisphosphonates," Current Opinion in Pharmacology, vol. 6, no. 3, pp. 307312, 2006.

[57] M. L. Tiku, J. B. Liesch, and F. M. Robertson, "Production of hydrogen peroxide by rabbit articular chondrocytes. Enhancement by cytokines," Journal of Immunology, vol. 145, no. 2, pp. 690-696, 1990.

[58] K. Bauerova and A. Bezek, "Role of reactive oxygen and nitrogen species in etiopathogenesis of rheumatoid arthritis," General Physiology and Biophysics, vol. 18, pp. 15-20, 1999.

[59] R. Serretti, P. Core, S. Muti, and F. Salaffi, "Influence of dichloromethylene diphosphonate on reactive oxygen species production by human neutrophils," Rheumatology International, vol. 13, no. 4, pp. 135-138, 1993.

[60] E. J. Dombrecht, C. B. De Tollenaere, K. Aerts et al., "Antioxidant effect of bisphosphonates and simvastatin on chondrocyte lipid peroxidation," Biochemical and Biophysical Research Communications, vol. 348, no. 2, pp. 459-464, 2006.

[61] P. Pelegrin and A. Surprenant, "Dynamics of macrophage polarization reveal new mechanism to inhibit IL-1beta release through pyrophosphates," The EMBO Journal, vol. 28, no. 14, pp. 2114-2127, 2009.

[62] G. Lopez-Castejón, A. Baroja-Mazo, and P. Pelegrín, "Novel macrophage polarization model: from gene expression to identification of new anti-inflammatory molecules," Cellular and Molecular Life Sciences, vol. 68, no. 18, pp. 3095-3107, 2010.

[63] H. Zimmermann, "Extracellular metabolism of ATP and other nucleotides," Naunyn-Schmiedeberg's Archives of Pharmacology, vol. 362, no. 4-5, pp. 299-309, 2000.

[64] A. M. Ho, M. D. Johnson, and D. M. Kingsley, "Role of the mouse ank gene in control of tissue calcification and arthritis," Science, vol. 289, no. 5477, pp. 265-270, 2000.

[65] S. C. Sun and S. C. Ley, "New insights into NF- $\kappa$ B regulation and function," Trends in Immunology, vol. 29, no. 10, pp. 469$478,2008$.
[66] G. Gloire and J. Piette, "Redox regulation of nuclear posttranslational modifications during NF- $\kappa$ B activation," Antioxidants and Redox Signaling, vol. 11, no. 9, pp. 2209-2222, 2009. 


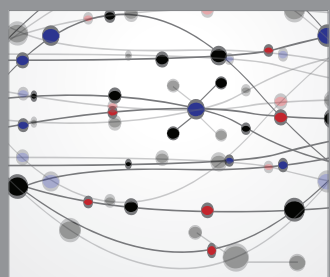

The Scientific World Journal
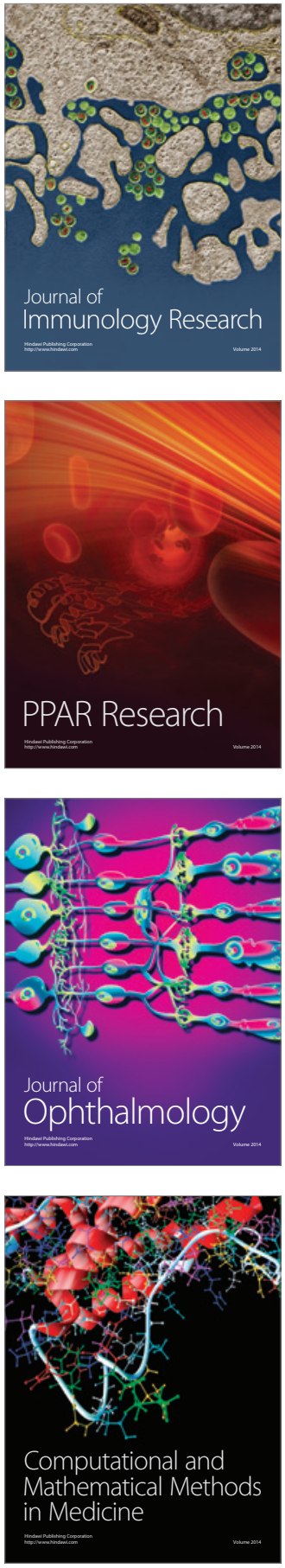

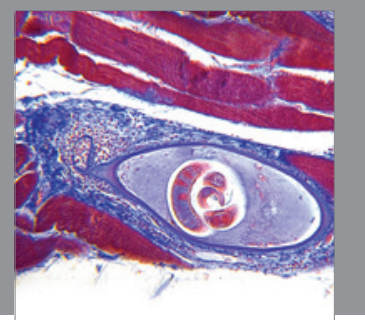

Gastroenterology

Research and Practice
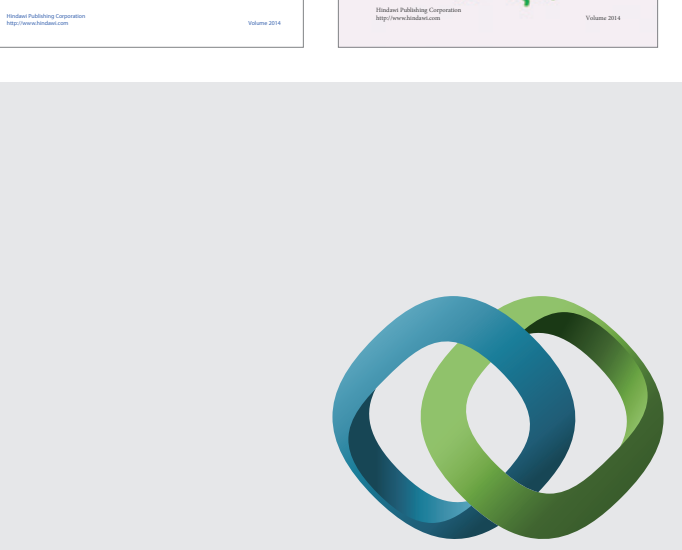

\section{Hindawi}

Submit your manuscripts at

http://www.hindawi.com
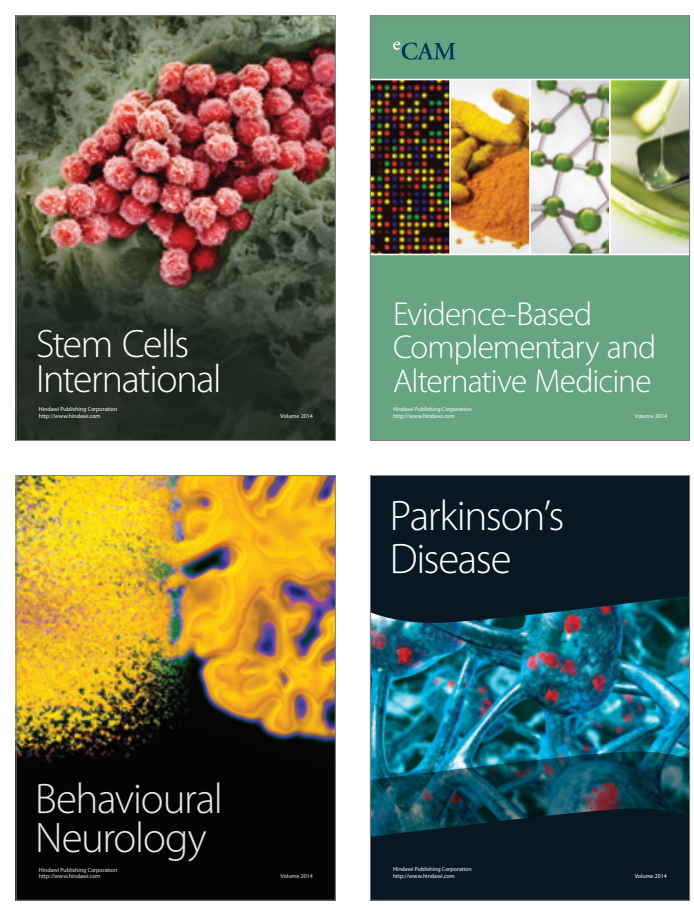

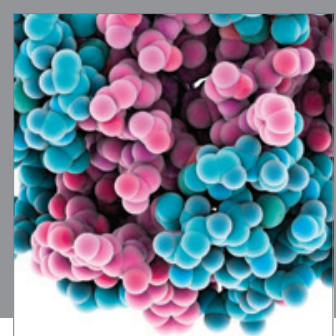

Journal of
Diabetes Research

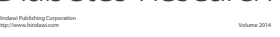

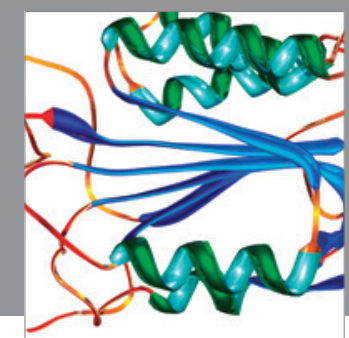

Disease Markers
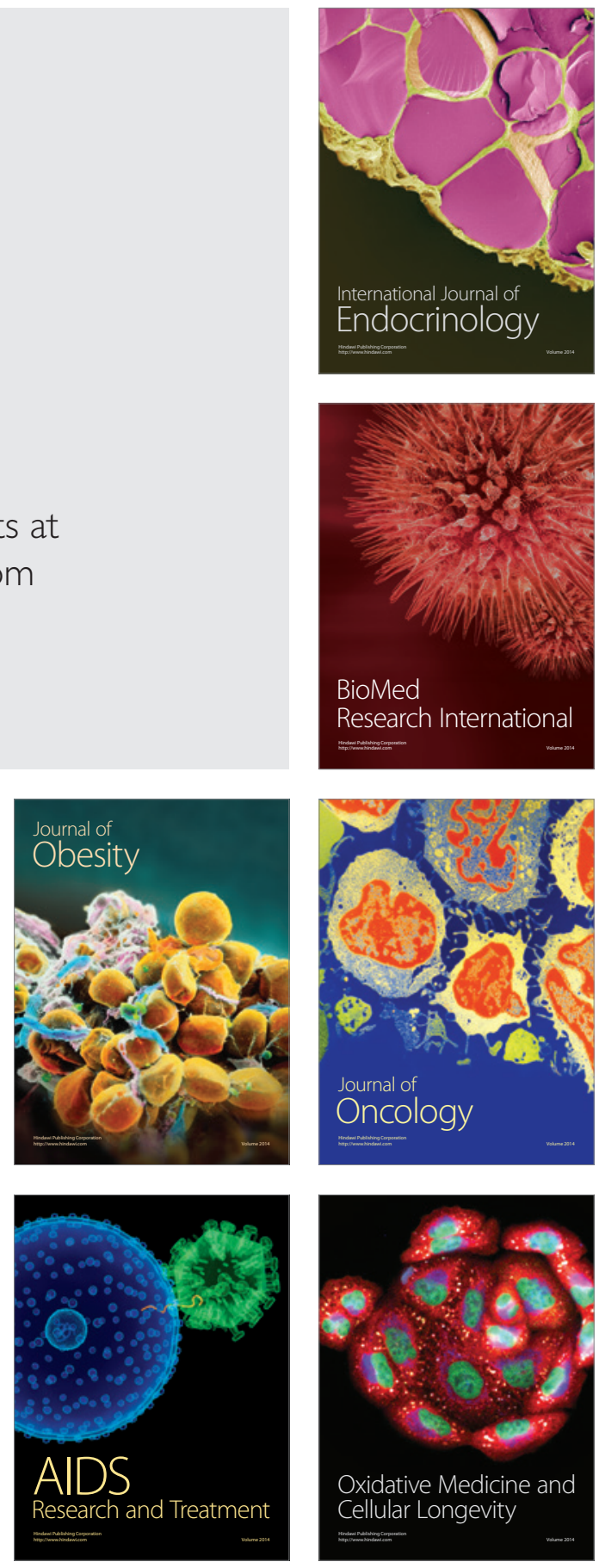